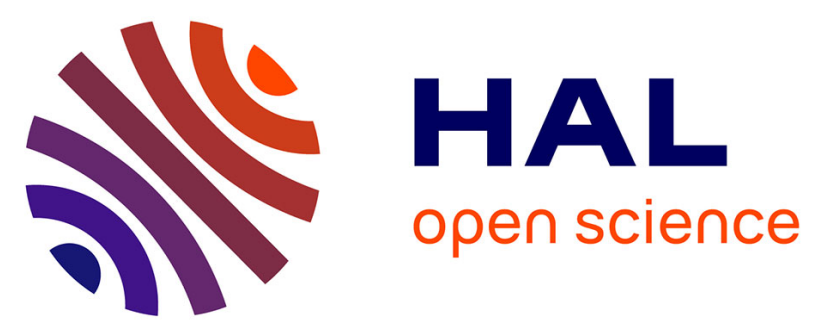

\title{
Innovative in cellulo method as an alternative to in vivo neurovirulence test for the characterization and quality control of human live Yellow Fever virus vaccines: a pilot study
}

Anaelle da Costa, Christophe Prehaud, Cécile Khou, Nathalie Pardigon, Aure Saulnier, Nolwenn Nougarede, Monique Lafon

\section{To cite this version:}

Anaelle da Costa, Christophe Prehaud, Cécile Khou, Nathalie Pardigon, Aure Saulnier, et al.. Innovative in cellulo method as an alternative to in vivo neurovirulence test for the characterization and quality control of human live Yellow Fever virus vaccines: a pilot study. Biologicals, 2018, 10.1016/j.biologicals.2018.03.004 . pasteur-01783750

\section{HAL Id: pasteur-01783750}

https://hal-pasteur.archives-ouvertes.fr/pasteur-01783750

Submitted on 2 May 2018

HAL is a multi-disciplinary open access archive for the deposit and dissemination of scientific research documents, whether they are published or not. The documents may come from teaching and research institutions in France or abroad, or from public or private research centers.
L'archive ouverte pluridisciplinaire HAL, est destinée au dépôt et à la diffusion de documents scientifiques de niveau recherche, publiés ou non, émanant des établissements d'enseignement et de recherche français ou étrangers, des laboratoires publics ou privés. 
Innovative in cellulo method as an alternative to in vivo neurovirulence test for the characterization and quality control of human live Yellow Fever virus vaccines: a pilot study

Anaelle da Costa $^{\mathrm{a}, \mathrm{b}^{*}}$, Christophe Prehaud ${ }^{\mathrm{a}^{*}}$, Cecile Khou ${ }^{\mathrm{c}}$, Nathalie Pardigon ${ }^{\mathrm{c}}$, Aure Saulnier $^{\mathrm{b}}$, Nolwenn Nougarede ${ }^{\mathrm{b}}$, Monique Lafon ${ }^{\mathrm{a}}$

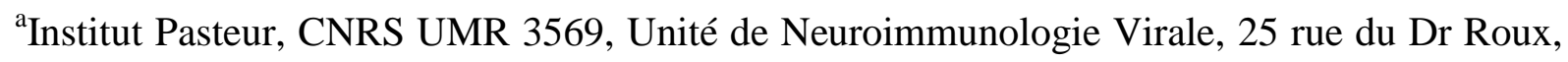
75724 Paris Cedex 15, France ; ${ }^{\mathrm{b}}$ Sanofi Pasteur, Plateforme de caractérisation immunologique et microbiologique, Département analytique de la R\&D, 1541 Avenue Marcel Mérieux, 69280 Marcy l'Etoile, France; 'Institut Pasteur, Unité de recherche et d'expertise Environnement et risques infectieux, Groupe Arbovirus, 25 rue du Dr Roux, 75724 Paris Cedex 15, France

* Co-first authors

\# Corresponding author: Monique Lafon

Tel : +3314568 8752 ; E-mail: monique.lafon@pasteur.fr

Running title: Novel in cellulo test of live YFV vaccines

Word count: Abstract: 192, Full text: 5551 


\begin{abstract}
Live attenuated vaccines have proved to be mostly valuable in the prevention of infectious diseases in humans, especially in developing countries. The safety and potency of vaccine, and the consistency of vaccine batch-to-batch manufacturing, must be proven before being administrated to humans. For now, the tests used to control vaccine safety largely involve animal testing. For live viral vaccines, regulations require suppliers to demonstrate the absence of neurovirulence in animals, principally in non-human primates and mice. In a search to reduce the use of animals and embracing the 3Rs principles (Replacement, Reduction, Refinement in the use of laboratory animals), we developed a new Blood-Brain Barrier Minibrain (BBB-Minibrain) in cellulo device (i.e. a device used to conduct experiments with cells outside the organism (animal) and not in glassware) $[1,2]$ to evaluate the neuroinvasiveness/neurovirulence of live Yellow Fever virus (YFV) vaccines. A pilot study was performed using the features of two distinct YFV strains, with the ultimate goal of proposing a companion test to characterize YFV neurovirulence. Here, we demonstrate that the BBB-Minibrain model is a promising alternative to consider for future replacement of YFV vaccine in vivo neurovirulence testing (see graphical abstract).
\end{abstract}

Keywords: 3Rs (Replacement, Reduction, Refinement), vaccine, Yellow Fever virus, nonhuman primates, BBB-Minibrain, in cellulo model 


\section{Introduction}

Vaccination is one of the most cost-effective health investments, with proven strategies to protect from infectious diseases and increase the live expectancy of humans. Vaccines are sometimes prepared using live non-virulent viruses [3]. Their efficacy and the need for limited injections compared to inactivated vaccines make them useful tools. Before becoming licensed for human immunization, the seeds of live viral vaccines, such as those of Yellow Fever virus (YFV), Measles or Poliovirus need to be tested for efficacy and safety, including monitoring for the absence of neurovirulence [4, 5]. Currently, safety testing is mainly performed in animal models[6, 7]. There are a number of reasons to seek alternatives to this, namely; the cost of animal studies, the difficulties associated with measuring pathology in animal brains, and the ethical questions regarding the use of animals in experimentation, particularly non-human primates. All these reasons are strong drivers for the development of

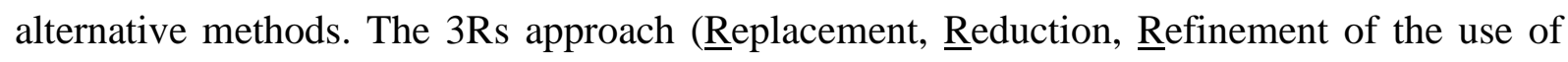
animals in scientific research) is clearly a priority for health authorities globally, and poses a challenge for scientists to develop alternative in cellulo tests that obviate the need for animal experimentation [8-12]. Cellular tests, which could amplify and detect the neurovirulence phenotypic traits of live vaccines, would be highly desirable. Virus-mediated neurological disease entails quite complex polygenic traits, requiring the acquisition of neuroinvasiveness (entry into the central nervous system [CNS]), by passage for instance through the bloodbrain barrier (BBB), neurotropism (the capacity to infect neural cells and propagate in the nervous system), and virus-induced neuronal homeostasis perturbation (neurovirulence) [13, 14]. The growth, within the CNS, of a pathogenic viral population could be the result of it acquiring neuropathogenic properties that originate from fixation of a few genomic mutations [15]. Moreover, neurological disorders (i.e. neuroinflammation, neurotoxicity, perturbation of 
neuronal homeostasis) result in the modulation of distinct clusters of cellular genes, activated by neuropathogenic viruses and not by their non-neurovirulent counterparts $[16,17]$.

Cerebral endothelial cells, forming tight and adherent junctions, constitute the main anatomical basis of the $\mathrm{BBB}$. There is a dynamic interaction between the brain endothelium, other neighboring cells (such as astrocytes, pericytes, perivascular microglia) and neurons which are pivotal to maintaining the integrity of the BBB. The three major functions of the $\mathrm{BBB}$ are the creation and maintenance of ionic homeostasis for neural functions, the supply of the CNS with nutrients, and protection from exogenous toxic injuries including those inflicted by pathogens $[18,19]$. Some neurotropic viruses (e.g. HTLV, AAV9, HIV, WNV) have acquired neuroinvasive capacity, that is to directly or indirectly cross the BBB [20,21].

Our objective was to design an in cellulo test capable of monitoring the absence of neurovirulence of a live YFV vaccine batch. Such a test must be highly sensitive and capable of firstly, revealing rare viral neuroinvasive/neurovirulent emerging events that enable the virus to enter the brain through the BBB; secondly, amplify the mutant population in a model, mimicking brain parenchyma, after its passage through the BBB; and finally, be able to detect specific features of the neuroinvasive/neurovirulent mutants.

We recently designed and developed a BBB-Minibrain culture device (da Costa et al., in preparation), which appears potentially suitable for such a test. The device consists of a in cellulo model of BBB formed by endothelial cell (hCMEC/D3)-coated Transwells ${ }^{\circledR}$ porous filters and cell culture wells, containing a mixed culture of human brain cells (neurons, astrocytes and microglia) called Minibrain (Fig 1A). The Minibrain tri-culture system thus mimics a simplified cerebral parenchymal environment, with the exception of oligodendrocytes. We anticipate this device responding to all three requirements; selecting and amplifying rare variants exhibiting neuroinvasive/neuropathogenic properties, and 
detecting homeostasis perturbations induced by the neuroinvasive/neurovirulent mutants in the sub-compartment containing the Minibrain.

We assessed the BBB-Minibrain culture device as an in cellulo test, to isolate and amplify rare neuroinvasive/neurovirulent variants potentially present in YFV strains. Two YF live vaccine strains were included in this study: 17D strain and the French Neurotropic Virus (FNV) strain. They were obtained by passaging with Asibi and French Viscerotropic Virus (FVV) strains, respectively, in the mid-1930s [22-24]. The 17D vaccine is a safe, and efficacious, live-attenuated vaccine still in use today [24]. FNV, although slightly more efficacious than 17D, was found to cause post-vaccination neuropathogenesis in children (0.3-0.4\%), and thus was discontinued in $1980[22,23]$. We chose this pair of YFV vaccine strains for our test : the FNV strain as a prototype of neuroinvasive/neurovirulent YF viruses, and 17D strain as a prototype of non-neuroinvasive/neurovirulent virus [25]. We compared the capacity of FNV and 17D viruses to cross the BBB, in the BBB-Minibrain model, we assessed their ability to infect the Minibrain, multiply in it, and perturbate its homeostasis. Our results demonstrate that it is feasible to detect neurovirulent variants, reliably and timely in cellulo with the BBB-minibrain. This system represents a promising development, and could ultimately enable the replacement of animals for neurovirulence testing of vaccine from which live virions can cross the BBB. 


\section{Material and methods}

\subsection{Cells}

hCMEC/D3 cells are human cerebral microvessel endothelial cells [26]. The cells were purchased from Tebu-Bio (France). They were maintained at $37^{\circ} \mathrm{C}$ on rat collagen $(0.1 \mathrm{mg} / \mathrm{ml}$ in water, Cultrex, 3443-100-01, R\&D Systems, U.K.) in EndoGro medium (Merck Millipore, SCME004, France) supplemented with $5 \%$ fetal bovine serum (FBS) and 1\% penicillinstreptavidin (Thermofisher, 15140, France).

For experiments, $5.10^{4}$ or $2.10^{5}$ hCMEC/D3 cells were seeded onto collagen-coated cell culture Transwell $^{\circledR}$ inserts, 12- or 6-well plate inserts, respectively (Corning, 3460; Corning, 3450, USA) and grown for 6 days. Under these conditions, hCMEC/D3 cells formed a confluent monolayer after 3 days and formed a barrier, with tight junctions, after 6 days [26].

NT2-N/A, a mixed culture of neurons and astrocytes [17, 27], was differentiated from Ntera2clD/1 cells (ATCC CRL1973), as described elsewhere [28, 29]. A tri-culture was obtained by adding human CHME microglial cells [30] at a 1:10 ratio. Phase-contrast microscopy pictures of Figure S1A show Ntera-2clD/1 (upper panel), NT2-N/A, mixed culture of neurons and astrocytes (middle panel) and CHME, microglial cells (bottom panel). Of note, the CHME we used were phenotyped to prove that they were of human origin (Homo sapiens CCNT1 phenotyping) and not of rat (Rattus norvegicus) origin as claimed by Garcia-Mesa Y. et al. [31]. This tri-culture, called Minibrain, was maintained at $37^{\circ} \mathrm{C}$ in Dulbecco's Modified Eagle Medium/Nutrient Mixture F-12 (DMEM-F12) (Thermofisher, 31330, France) supplemented with 5\% FBS (EuroBio, CVFSVF01-01, France), 1\% L-glutamine (Thermofisher, 25030, France) and 1\% penicillin-streptavidin (Thermofisher, 15140, France). 
African green monkey kidney cells Vero (ATCC CRL-1586, positive control cell line for Yellow Fever viruses) were maintained at $37^{\circ} \mathrm{C}$ in DMEM supplemented with 5\% FBS and $1 \%$ penicillin-streptavidin.

\section{2. hCMEC/D3-Minibrain co-culture}

For the co-culture experiments between hCMEC/D3 on inserts, and Minibrain in the cell culture well, the hCMEC/D3 and Minibrain cells were seeded the same day, on inserts or wells respectively, in EndoGro medium supplemented with 5\% FBS and 1\% penicillinstreptavidin. Each day, during the 6 days of culture needed by the hCMEC/D3, inserts containing hCMEC/D3 cells were placed in wells containing Minibrain cells. On the sixth day, the paracellular permeability of hCMEC/D3 was tested for each condition by monitoring the permeability to lucifer yellow (LY).

\subsection{Assessment of the restrictive paracellular permeability with LY}

The restrictive paracellular permeability of hCMEC/D3 was assessed by their low permeability to the non-permanent fluorescent LY (Sigma Aldrich, L0259, USA).

Briefly, for 12-well inserts, hCMEC/D3 monolayers cultivated for 6 to 7 days on inserts, were transferred to 12 -well plates containing $1.5 \mathrm{~mL}$ of transport medium [(HBSS, Thermofisher, 14025-100, France) supplemented by $10 \mathrm{mM}$ of hepes (Thermofisher, 15630-080, France) and $1 \mathrm{mM}$ of sodium pyruvate (Thermofisher, 11360)] per well (abluminal compartment). Transport medium $(0.5 \mathrm{~mL})$ containing $50 \mu \mathrm{M}$ of $\mathrm{LY}$ was then added to the luminal compartment. Incubations were performed at $37^{\circ} \mathrm{C}, 5 \% \mathrm{CO}_{2}, 95 \%$ humidity. After 10,25 and $45 \mathrm{~min}$, the inserts were transferred into new wells, previously filled with $1.5 \mathrm{~mL}$ of transport medium. After $45 \mathrm{~min}$, aliquots were taken for each time point, from both compartments and 
the concentration of LY determined using a fluorescence spectrophotometer (Tecan Infinite F500, USA). For 6-well inserts, the same method was used with $1.5 \mathrm{~mL}$ in the luminal compartment and $2.5 \mathrm{~mL}$ in the abluminal compartment.

The endothelial permeability coefficient $\left(\mathrm{P}_{\mathrm{e}}\right)$ of LY was calculated in centimeters/min $(\mathrm{cm} / \mathrm{min})$, as described by Siflinger-Birnboim et al. [32]. To obtain a concentrationindependent transport parameter, the clearance principle was used. Briefly, the average volume cleared was plotted versus time, and the slope was estimated by linear regression. Both insert permeability $\left(\mathrm{PS}_{\mathrm{f}}\right.$, for insert only coated with collagen) and insert plus endothelial cell permeability $\left(\mathrm{PS}_{\mathrm{t}}\right.$, for insert with collagen and cells) were taken into consideration, according to the following formula: $1 / \mathrm{PS}_{\mathrm{e}}=1 / \mathrm{PS}_{\mathrm{t}}-1 / \mathrm{PS}_{\mathrm{f}}$. The permeability value for the endothelial monolayer was then divided by the surface area of the porous membrane of the insert to obtain the endothelial permeability coefficient $\left(\mathrm{P}_{\mathrm{e}}\right)$ of the molecule (in $\left.\mathrm{cm} / \mathrm{min}^{-1}\right)$. As a quality control of our experiments, we considered that a Pe higher than 1.2 would mean a defect in the BBB integrity [33, 34].

\subsection{Virus stock production}

YFV strain 17D (Gene Bank MG051217) and YFV strain FNV (From the collection of Institut Pasteur Dakar, Senegal, Gene Bank MG051218) were produced in Vero cells. To produce viral stocks, Vero cells grown in EndoGro medium supplemented with $2 \%$ FBS were infected with the viruses at a multiplicity of infection (MOI) of 0.1 and supernatants were harvested 4 days post-infection at the onset of cytopathic effect. Supernatants were then centrifuged for $5 \mathrm{~min}$ at $1200 \mathrm{~g}$ and stored at $-80^{\circ} \mathrm{C}$. Viral titers obtained for $17 \mathrm{D}$ and FNV were not significantly different (Figure S1B). 


\subsection{Virus titration by plaque assay}

Vero cells were seeded in 24-well plates. Tenfold dilutions of virus samples were prepared in DMEM and $200 \mu \mathrm{L}$ of DMEM supplemented with $2 \%$ FBS and $200 \mu \mathrm{L}$ of each dilution were added to the cells. The plates were incubated for $90 \mathrm{~min}$ at $37^{\circ} \mathrm{C}$. Unadsorbed viruses were removed, $400 \mu \mathrm{L}$ of DMEM supplemented with $2 \%$ FBS and $400 \mu \mathrm{L}$ of DMEM supplemented with $1.6 \%$ carboxymethyl cellulose (CMC), $10 \mathrm{mM}$ HEPES buffer, $72 \mathrm{mM}$ sodium bicarbonate, and $2 \% \mathrm{FBS}$ were added to each well, followed by incubation at $37^{\circ} \mathrm{C}$ for 5 days. The CMC overlay was eliminated, and the cells were washed with PBS, fixed and stained with $4 \%$ paraformaldehyde/5\% crystal violet for 20 min and rinsed 3 times with water. The titers are expressed in plaque forming unit $(\mathrm{pfu}) / \mathrm{mL}$

\subsection{Viral production kinetic on Minibrain cells}

Minibrain cells grown in 24-well plates in EndoGro medium supplemented with 2\% FBS were infected with YFV strains $17 \mathrm{D}$ or FNV at a MOI of 0.5 . The plates were incubated for 2 $\mathrm{h}$ at $37^{\circ} \mathrm{C}$. Unadsorbed virus particles were removed, and EndoGro medium supplemented with $2 \%$ FBS was added. Supernatant was harvested and frozen at different time points $(0,24$, 48 and $72 \mathrm{~h}$ ), and then titrated as described above.

\subsection{Cellular marker expression quantification by qRT-PCR}

Minibrain total RNAs were extracted and purified with QIAshredder column (Qiagen, 79656, Germany) and RNeasy minikit (Qiagen, 74104, Germany) following the manufacturer's instructions. RNA quantity and quality were monitored using spectrophotometry (NanoDrop, USA). Reverse transcription-PCR (RT-PCR) was performed using a PX2 thermal cycler. 
cDNA synthesis was performed from $0.36 \mu \mathrm{g}$ total RNA, using SuperScript II reverse transcriptase (Thermofisher, 18064, France) according to the manufacturer's instructions.

Quantitative real-time PCR (qPCR) was performed in triplicate using an ABI Prism 7500 fast sequence detector system with Go Taq master mix (Promega, A6001, France), with 30 cycles of amplification. After normalization to $18 \mathrm{~S}$ RNA, the relative abundance of mRNA was obtained by calculation of the difference in threshold cycles of the test and control sample (Caco2 cells RNA), commonly known as the $\Delta \Delta \mathrm{CT}$ method. CD200R (QT00037569) and TH (QT00067221) primers were purchased from Qiagen (Germany). Primers for 18S: 5'CTtAGAGGGACAAGTGGCG-3' and 5'-ACGCTGAGCCAGTCAGTGTA-3'. Primers for aquaporin $\quad 4: \quad$ 5'-GGTATAGTCAATTCTTATTTGAAT-3' and 5'CTTGAATCTCAATAGGTGCCCTTA-3'. Primers for GFAP: 5'CTGCTTCTTAACCCCAGTAAGCCA-3' and 5'-CAGCAGTGCCCTGAAGATTAGCAG3'. Primers for PYGB: 5'-TCCTGCTGTGTCCTGAGGTGCATT-3' and 5'GCCCAGATCCAGCATGCAAGGTGC-3'. Primers for NEFH: 5'GATGAAGATTGAGCGGCCTGTAAC-3' and 5'-TCCTCCGCTTCCGCTGCACCTCTT3 '.

\subsection{Immunostaining}

2.8.1. Staining of hCMEC/D3 and Vero cells grown on coverslips:

After $24 \mathrm{~h}$ of infection at a MOI of 0.1 , cells were fixed with $4 \%$ formaldehyde for $10 \mathrm{~min}$ followed by permeabilization with $0.2 \%$ Triton $\mathrm{X}-100$ for 2 min. After permeabilization, the cells were washed with PBS, and incubated for $1 \mathrm{~h}$ at $37^{\circ} \mathrm{C}$ with PBS-milk 5\%. The cells were incubated for $1 \mathrm{~h}$ at $37^{\circ} \mathrm{C}$ either with anti-Flavivirus E protein mouse antibody (4G2) (Biotem,

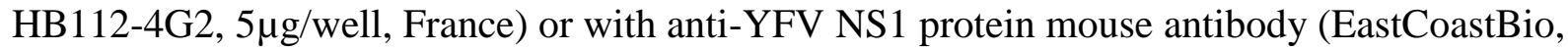
$\mathrm{HM} 489,2 \mu \mathrm{g} / \mathrm{well}, \mathrm{USA})$ in PBS-milk $2 \%$, followed by incubation for $1 \mathrm{~h}$ at $37^{\circ} \mathrm{C}$ with Alexa 
Fluor 488-conjugated anti-mouse IgG antibody (Invitrogen, A11001, 1/2000, France) in PBSmilk $2 \%$. The cells were then incubated for $30 \mathrm{~min}$ at $37^{\circ} \mathrm{C}$ with Cellmask (Molecular probes, C10045, USA) in PBS-milk 2\%. The coverslips (Merck-Millipore, PEZGS0416, France) were mounted with ProLong gold antifade reagent with DAPI, a nucleus marker (Thermofisher, P36935, France). The slides were examined using a fluorescence microscope (Axio observer D1, Zeiss, Germany).

\subsubsection{NT2-N cell staining:}

After $48 \mathrm{~h}$ of infection at a MOI of 1, cells were fixed with $4 \%$ paraformaldehyde for 20 min followed by permeabilization with $0.5 \%$ Triton X-100 for $10 \mathrm{~min}$. The cells were then washed with PBS, and incubated overnight at $4{ }^{\circ} \mathrm{C}$ with PBS with $50 \%$ goat serum. Then, the cells were incubated overnight at $4^{\circ} \mathrm{C}$ with anti-NF200 (neuronal neurofilament) rabbit antibody (Sigma, N4142, 1/1000, USA) and anti-Flavivirus E protein mouse antibody (4G2) (R\&D biotech, 1/1000, USA) in PBS with 50\% goat serum, followed by incubation for $1 \mathrm{~h}$ at $37^{\circ} \mathrm{C}$ with Alexa Fluor 488-conjugated anti-rabbit IgG (Invitrogen, A11034, 1/500, France) and Cy3-conjugated anti-mouse IgG antibody (Jackson, 715-165-150, 1/500, USA) and Hoescht (Sigma, 33263, 1/200, USA) in PBS with 50\% goat serum. The coverslips were mounted with ProLong gold antifade reagent (Thermofisher, P36930, France). The slides were examined using a fluorescence microscope (LSM 700, Zeiss, Germany). The percentage of infected neurons was calculated by screening $>50$ fields in each condition.

\subsection{YFV RNA quantitation by RT-ddPCR}

Minibrain RNAs were extracted and purified with QIAshredder column (Qiagen, 79656, Germany) and RNeasy minikit (Qiagen, 74104, Germany) following the manufacturer's instructions. For the RT-droplet digital PCR (RT-ddPCR) assay, $5 \mu \mathrm{L}$ of tenfold dilutions of total RNA extract was combined with $20 \mu \mathrm{L}$ of master mix (Bio-Rad, 186-4021, USA), 
primers (5'-GCACGGATGTAACAGACTGAAGA-3' and 5'-CCAGGCCGAACCTGTCAT3') and probe (5'FAM-CGACTGTGTGGTCCGGCCCATC-3'BHQ1) and then placed in a well in the 8-well droplet generator cartridge (BioRad, Bio-Rad, 186-4108, USA) with $70 \mu \mathrm{L}$ of droplet generator oil (BioRad, 186-4110, USA). The cartridge was placed in the droplet generator machine (BioRad, USA). The droplets were transferred to a PCR plate which was placed in a T100 PCR thermal cycler apparatus (BioRad, USA) with the following program: reverse transcription step at $50^{\circ} \mathrm{C}(60 \mathrm{~min})$, followed by an initial PCR activation step at $95^{\circ} \mathrm{C}$ (15 min), 45 cycles of denaturation at $94^{\circ} \mathrm{C}\left(30 \mathrm{sec}\right.$, ramping $\left.2.5^{\circ} \mathrm{C} / \mathrm{sec}\right)$, annealing and extension at $60^{\circ} \mathrm{C}\left(60 \mathrm{sec}\right.$, ramping $\left.2.5^{\circ} \mathrm{C} / \mathrm{sec}\right)$ and a final step of enzyme heat kill at $98^{\circ} \mathrm{C}(10$ min). Fluorescence in droplets was measured using the QX100 Droplet Reader (BioRad, USA). Droplets were considered positive when the specific fluorophore (FAM: 6carboxyfluorescein) was detected. The results were analyzed to determine the concentration of sample, expressed as genome equivalent (Geq) per $\mu \mathrm{g}$ of total RNA.

\subsection{Evaluation of YFV neuroinvasive phenotype (capacity to pass though the BBB)}

hCMEC/D3 cells were seeded in inserts in EndoGro medium supplemented by 5\% FBS 6 days prior to the virus inoculation (H0). EndoGro medium supplemented by $2 \%$ FBS was substituted to the original medium $24 \mathrm{~h}$ before the onset of the experiment. At the onset of the experiment (H0), $50 \mu \mathrm{L}$ (corresponding to $3.5 \times 10^{3} \mathrm{pfu} / \mathrm{insert}$ ) of the viruses produced in EndoGro medium supplemented with $2 \%$ FBS were added in the luminal compartment. $24 \mathrm{~h}$ later, the inserts containing hCMEC/D3 cells were removed and tight junction integrity was assessed by measuring of the endothelial permeability to LY. RNAs were extracted for transcriptomic studies. The abluminal compartment supernatant was used to quantify the viruses by titration. 


\subsection{Evaluation of YFV neuroinvasive and neurotropic phenotypes (capacity to pass}

through the BBB and multiply in the Minibrain, respectively)

hCMEC/D3 cells were seeded in inserts in EndoGro medium supplemented by 5\% FBS 6 days prior to the virus inoculation (H0). Minibrain cells were seeded at the bottom of the wells in DMEM supplemented with $5 \%$ of FBS 4 days prior to the virus inoculation. hCMEC/D3 and Minibrain cells were co-cultured in EndoGro medium supplemented by 2\% FBS $24 \mathrm{~h}$ before the onset of the experiment. At $\mathrm{H} 0,50 \mu \mathrm{L}$ of the viruses produced in EndoGro medium supplemented with $2 \%$ FBS were added in the upper compartment (corresponding to $3.5 \times 10^{3} \mathrm{pfu} / \mathrm{insert}$ ). The inserts containing hCMEC/D3 cells were removed $24 \mathrm{~h}$ later and tight junction integrity was assessed by measuring the endothelial permeability to LY. The Minibrain supernatant was used to quantify the viruses in the lower abluminal compartment by titration and medium was renewed.

$48 \mathrm{~h}$ later (H72), the Minibrain supernatant was used to quantify the viruses in the abluminal compartment by titration and the Minibrain RNAs were extracted to quantify the viral RNA in the Minibrain cells by ddPCR and/or for transcriptomic studies.

\subsection{Transcriptomic studies}

RNAs were extracted and purified with QIAshredder column (Qiagen, 79656, Germany) and RNeasy plus minikit (Qiagen, 74134, Germany) according to the manufacturer's instructions from 24 h-infected hCMEC/D3 or 72 h-infected Minibrain. Transcriptomic experiments were sub-contracted to CERBM (Plateform GenomEast of IGBMC, Strasbourg, France) and performed using Affymetrix GeneChip Human Genome U133 2.0 Plus. Briefly, biotinylated complimentary RNA (cRNA) targets were prepared using the "MessageAmp" Amplification Kit" from Ambion (USA), according to the Instruction Manual (\# 4386269 Revision B, 18 September 2007), starting from 300 ng of total RNA. 
Following fragmentation, $10 \mu \mathrm{g}$ of cRNAs were hybridized for $16 \mathrm{~h}$ at $45^{\circ} \mathrm{C}, 60 \mathrm{rpm}$ on Human GeneChip ${ }^{\circledR}$ HG-U133 plus 2.0 arrays (Affymetrix, USA). The chips were washed and stained in the GeneChip ${ }^{\circledR}$ Fluidics Station 450 (Affymetrix, USA) using the FS450_0004 script and scanned with the GeneChip ${ }^{\circledR}$ Scanner 3000 7G (Affymetrix, USA) at a resolution of $1.56 \mu \mathrm{m}$. Raw data (CEL Intensity files) were extracted from the scanned images using the Affymetrix GeneChip ${ }^{\circledR}$ Command Console (AGCC) version 4.1.2 (Affymetrix, USA).

CEL files were further processed with Affymetrix Expression Console software version 1.4.1 to calculate probeset signal intensities, using Robust Multi-array Average (RMA) algorithms with default settings. A cut off of 2.1 fold change at $\mathrm{p}<0.05$ (multiple Student's t test) was used to cluster the genes. Genes with significant fold change were compiled and data mined by using the ingenuity pathway analysis software and data banks (www.ingenuity.com, a Qiagen Company, USA). Networks analysis identifies key regulatory networks and pathways that are perturbed and then determines which plausible upstream regulator could cause the resulting changes in gene expression. The major biological functions, the transcription regulators and signaling pathways affecting these genes were determined (Fisher's Exact Test p-value $<0.05-0.01)$.

\subsection{Statistical analyses}

Statistical analyses were performed using GraphPad Prism 7.03. Unpaired Student's t test two tailed were used as statistical tests. 


\section{Results}

\subsection{The BBB-Minibrain, an in cellulo model to study the neurovirulence of YF vaccine strains}

The Minibrain, a tri-culture of co-differentiated neurons and astrocytes (NT2-N/A) [17, 27] and microglial cells (CHME) [30], was developed as a model of brain parenchyma, to evaluate the virus neurovirulence. To mimic both the BBB and the brain parenchyma, a compartmentalized culture system was used, with Transwell ${ }^{\circledR}$ insert, which enabled the separation of the luminal ("blood") compartment on the upper side of the insert, and the abluminal ("brain") compartment in the well under the insert (Figure 1A, left).

In this system, the hCMEC/D3 endothelial barrier permeability to $\mathrm{LY}\left(\mathrm{Pe}_{\mathrm{LY}}\right)$ neither increased nor decreased in the presence of the Minibrain, whatever the co-culture time point (Days 1-6, Figure 1A, right). This result clearly demonstrates that the co-culture system did not induce changes in hCMEC/D3 paracellular junctions, and the hCMEC/D3 barrier physical integrity.

The hCMEC/D3-BBB cell permissivity to 17D and FNV (i.e. the ability to infect, to replicate and to produce virus progeny) was monitored by immunofluorescence (Figure 1B). The two viruses (same batch and same amount of viral inoculum) can infect Vero cells (i.e. susceptible cell line for Yellow Fever viruses) as shown by the accumulation of viral antigens (E protein detection, Figure 1B, a \& b). Conversely, the accumulation of viral antigens (E protein detection) was not detected in the hCMEC/D3 endothelial cells (Figure 1B, c \& d) 24 h after addition of the virus particles. In order to improve the sensitivity of the detection of the replicative virus in $\mathrm{BBB}$, the infected cells were stained by an anti-NS1 antibody [35]. Nevertheless, the result was identical (Figure 1B, e $\& \mathbf{f}$ versus $\mathbf{g} \boldsymbol{\&} \mathbf{h}$ ). This confirms that hCMEC/D3 cells, as a physiological barrier, are not permissive to 17D and FNV. 
The cells in the abluminal Minibrain compartment were phenotyped by qRT-PCR (Figure 1C). The presence of neuronal (NEFH, TH), astrocytic (AQ4, PYGB, GFAP) and microglial (CD200R) markers was established by gene expression analysis (Figure 1C, right).

Then, the permissivity of the Minibrain-Neurons to the two virus strains was evaluated (Figure 1C, left). NT2-N neurons can be infected by both YFV stains (Figure 1D, a \& $\mathbf{c}$ for $17 \mathrm{D} ; \mathbf{b} \& \mathbf{d}$ for FNV) as demonstrated by the co-staining of the neuronal cells and the virus. The E detection pattern (red dot) is in agreement with the accumulation of E protein in the ER-Golgi network in neurons [36, 37]. To note, the dot staining was not observed in noninfected NT2-N cells (Figure S1C). These results show that FNV was able to infect 50\% of the neurons whereas 17D infected only 18\% (Figure 1D, right, upper graph), showing that FNV is more neuronotropic (ability to infect neurons) than 17D. Surprisingly, the production of infectious virions in the Minibrain cells was similar over time for both virus strains (Figure 1D, right lower graph), suggesting either that neurons may release more 17D virions or that cell types other than neurons may be infected by 17D viral strain.

\subsection{FNV is more neuroinvasive than 17D}

To monitor the neuroinvasive capacity of $17 \mathrm{D}$ and FNV, we firstly used the in cellulo BBB model alone, without Minibrain in the abluminal compartment. For this purpose, once the barrier was formed by the hCMEC/D3 cells [i.e. 6 days of culture on insert with BBB integrity checked by endothelial permeability to LY as described in Figure 1A)], the same MOI of each virus strain was added in the luminal compartment (Figure 2A). After removing the inserts $24 \mathrm{~h}$ later, $\mathrm{BBB}$ integrity was controlled (17D $\mathrm{Pe}_{\mathrm{LY}}=0.95+/-0.09$, FNV $\mathrm{Pe}_{\mathrm{LY}}=0.87+/-0.07$, N.I. (i.e. Mock infected) $\mathrm{Pe}_{\mathrm{LY}}=0.88+/-0.09$ ) and the infectious viral particles present in the abluminal compartment were titrated by plaque assay (Figure 2A). 
A higher quantity of infectious viral particles was detected with FNV as compared to 17D

(Figure 2B, Mean 17D=1, Mean FNV=5.5). Since there was no infection of confluent hCMEC/D3 by $24 \mathrm{~h}$ (Figure 1B), the difference of infectious virions in the lower compartment reflects a differential BBB passage of 17D and FNV. This shows that FNV is more neuroinvasive than $17 \mathrm{D}$ in this system. This is in agreement with the higher rate of neurotropic, post-vaccinal complications seen in children with the FNV vaccine strain $[24,38$, 39], indicating that the endothelial cells in the BBB-Minibrain device are able to differentiate YFV strains on the basis of their neuroinvasiveness.

\subsection{The neuroinvasive FNV virions are highly neurotropic}

We used the BBB-Minibrain compartmentalized culture model to study whether the neuroinvasive viral particles after "filtration" through the BBB could be amplified. Each virus was added at the same MOI in the luminal compartment, $24 \mathrm{~h}$ later the insert was removed, BBB integrity was assessed (17D $\mathrm{Pe}_{\mathrm{LY}}=0.88+/-0.04$, FNV Pe $\mathrm{LY}_{\mathrm{LY}}=0.95+/-0.06$, N.I. $\left.\mathrm{Pe}_{\mathrm{LY}}=0.82+/-0.06\right)$ and the viruses present in the abluminal compartment were titrated (Figure 2C). The infectious virus titers measured in the abluminal compartment, which originate from the Minibrain cells infected by the viral particles which have passed through the BBB, were higher with FNV than with 17D (Figure 2D, Mean 17D=1.1, Mean FNV=13.4). After the first $24 \mathrm{~h}$, the Minibrain cells were kept for an extra $48 \mathrm{~h}$ (Figure 2C). The viral progeny were then measured at $72 \mathrm{~h}$ by titration, by plaque assay (Figure 2E, Mean 17D=45.6, Mean FNV=1933.3) and RT-ddPCR (Figure 2F, Mean 17D=2.7e+04, Mean FNV=2.6e+06). With both methods, FNV titers were higher than 17D titers. The infectious viral titers were also higher at $72 \mathrm{~h}$ compared to $24 \mathrm{~h}$ for both YF strains, demonstrating the viral amplification of the neuroinvasive population in the cerebral cells. The overall titer difference between 17D and FNV was the same at $24 \mathrm{~h}$ and $72 \mathrm{~h}$, which is consistent with the 
identical viral production in Minibrain cells, as described earlier (Figure 1D, right lower graph) for both YF strains. These results indicate that the BBB-Minibrain can differentiate YF strains on the basis of their neurotropism.

\subsection{YFV neuroinvasiveness is correlated to a specific molecular signature}

In order to understand the mechanisms used by both YFVs to cross the BBB, we undertook a transcriptomic analysis of the endothelial cells $24 \mathrm{~h}$ after virus (or mock) passage (Figure 2A). Total RNA from the hCMEC/D3 cells were purified and used to identify the transcriptome on gene chips arrays, covering the entire human genome (Affymetrix, U133 plus 2). The data are presented in Figure 3. Despite the fact that both YF strains cannot infect the endothelial cells (Figure 1B), it can be seen from Figure 3A that the transcriptome of FNV and 17D treated cells were not neutral as compared to the mock infected cells. 17D triggered the up-regulation of 37 genes and FNV up-regulated 57 genes. Eighteen genes were stimulated by both 17D and FNV (Figure 3A and 3B). Interestingly, among this last cluster, were genes such as ALOX5, C1R, C1S, CEBPD, CHI3L1, FGG, HNRNPA1, HSD11B1, MAP3K8, SERPINF1 and SOCS3 (Figure 3B), these are well known to influence the differentiation and proliferation of cells and connective tissues. In order to determine which biological functions could be altered, we submitted this list of genes to a bioinformatic Intuitive Pathway Analysis (IPA, Qiagen, USA). 17D was found to slightly affect functions linked to cellular movement and development, as well as connective tissue disorders (Figure 3C, upper). This could explain why some 17D virions could cross the endothelial cells even though they are not able to infect and disrupt this barrier. Identically, FNV disabled the same cell functions but may have a stronger effect on endothelial cell biology as it impacts the expression of genes involved in cellular assembly and organization, as well as genes controlling cellular functions and their maintenance (Figure 3C, lower). Indeed, FNV can 
trigger the up-regulation of genes such as MTUS1, SMAD4 or XPO1, which are involved in cytoskeleton remodeling, as well as NAMPT, NBR1, SMAD4 and XPO1 which have been involved in autophagy (Figure 3B); a protective mechanism to avoid BBB breakage during stress $[40,41]$. This transcriptomic analysis suggests FNV is more efficient in modifying the endothelial cell barrier, leading to an increase in neuroinvasiveness. We are currently investigating how this gene regulation can be triggered, whether this can be linked to the existence of sub-viral species (i.e. quasispecies) of neuroinvasive variants, and what are the cellular paths exploited by these virions to cross the endothelial cells barrier.

\subsection{Towards a companion test based on transcriptomic analysis}

We showed that YF virions can cross the hCMEC/D3 barrier but their neuroinvasiveness depends on the origin of the vaccine strain. This led us to explore whether these viruses, which replicate with similar efficiency in the Minibrain, can trigger a specific molecular signature in the Minibrain. Total RNA of the Minibrain cells were purified at $72 \mathrm{~h}$ after virus (or mock) passage and used to identify the transcriptome, as described earlier. The data are presented in Figure 4. We found that both 17D and FNV vaccine strains stimulated the expression of 4243 and 4159 genes, respectively (at $\mathrm{p}<0.05$ ). No down-regulated genes were identified. This is in agreement with the data already obtained on human cohorts of patients vaccinated with YFV-17D vaccine, which exhibited only a strong up-regulation of gene expression in immune cells $[42,43]$. At a set-up of a 2.1 fold change, 17D triggered the expression of 30 different genes, contrary to FNV that stimulated the expression of 58 genes (Figure 4A). Interestingly, none of these genes were common between 17D and FNV. The list of genes is presented in Figure 4B. 17D stimulates a narrow cluster of genes which expression differs between 2.1 and 2.23 fold change); FNV stimulates a larger cluster (58 genes) but with a smaller variation in gene expression (2.1 up to 2.21 fold change, Figure 4B, 
pie diagrams) and with more genes grouped in the low fold change range (i.e. 2.1). It is interesting to note that both strains stimulated cerebral cell genes, which are known to play a role in hepatotoxicity (Figure 4B, shown in red). In order to identify the biological functions which can be altered by the neuroinvasive virions, we again submitted these two lists of genes to IPA (Figure 4C). We found that 17D virus strain affects the way the cells sense their local environment. This included cell movement and development, and also their interaction with other cells or the signaling pathways they sustained, whereas FNV virus strain altered the physiology of the cells by modifying their metabolism, or the transport and biochemistry of the molecules.

\section{Discussion}

Huge YF epidemics worldwide have been avoided, thanks to swift access of local health authorities to the manufactured live attenuated 17D YF vaccine, which is considered safe and efficient $[23,24,44]$.

17D live vaccines are derived from the Asibi virulent strain [45]. Empirical passages of Asibi virus strain for 76 passages in chick embryo tissues did not abolish the neurovirulent phenotype of the virus. A further 100 passages in cultures where the neurological tissues had been removed (i.e. minced chick embryo cells), created an attenuated YFV-176 strain [3]. The YF vaccine, which is derived from YF-204, is now manufactured in embryonic chicken eggs. 17D harbors 67 nucleotide differences, with Asibi encoding for 31 amino acids substitutions, and presents a very low quasispecies diversity [46]. Between 1938 and 1941, 17D vaccine batches were produced without strict control of passage levels. It was observed that some batches were over-attenuated and poorly immunogenic, whereas others were associated with post-vaccinal encephalitis. In 1941, it was decided to establish the "seed-lot" system, where 
passage level is highly controlled and primary seed and manufacturers working seed are prepared and characterized in non-human primates, for safety and immunogenicity [45]. The seed lot process adopted to manufacture the vaccine has proved to be reliable and safe [3]. The history of clinical records showing adverse events in humans, including neurovirulence, was made mandatory as a biological standard to enable careful evaluation of the safety of vaccine seeds, as stated by World health Organization (WHO) $[6,7,47]$. The current test for each virus master and working seed is undertaken in non-human primates (Macaca mulatta or Macaca fascicularis) to evaluate neurotropism, viscerotropism and immunogenicity [48, 49].

Ethical considerations questioning the use, and suffering, of animals in scientific experimentation, the cost of animal testing and the long-term difficulty of maintaining expertise for the interpretation of neurovirulence cerebral histopathology in animal models all support the need to find alternative methods $[50,51]$. In agreement with the $3 \mathrm{R}$ principles, we describe here a new in cellulo test, the BBB-Minibrain device, developed to limit animal testing in the field of viral live vaccine manufacturing. The system is based on a compartmentalized Transwell $^{\circledR}$ system with the upper part mimicking the BBB; with a layer of human endothelial cells and the lower part mimicking the brain with a cell monolayer of a triculture of human neuron, astrocyte and microglia. This system is biologically relevant and easy to set up and handle for professionals highly trained in cell culture [17, 27, 30, 52].

We used the BBB-Minibrain device to characterize the neuroinvasiness/neurovirulence of two prototype YFV strains (17D and FNV). We showed that 17D and FNV were unable to infect hCMEC/D3 when they constitute a biological barrier. However, both viruses were able to cross this barrier, FNV more efficiently than 17D. As FNV has previously been shown to be responsible for some vaccine neuropathogenesis [22], we hypothesize that this might be the result of FNV crossing the BBB more efficiently than 17D. Conversely, 17D, has been shown, although in rare circumstances, capable of crossing the BBB and causing encephalitis 
[53]. Our system has shown 17D has a very low crossing efficiency, this correlates strongly with experimental and clinical data [45]. Thus, the BBB-Minibrain device is able to discriminate a neuropathic vaccine strain (FNV) from a safe vaccine strain (17D). The transcriptomic analysis undertaken on the hCMEC/D3 barrier indicated that the "transit" of the YF 17D and FNV virions across the barrier had an impact on the endothelial cells. The fact that the alteration of BBB cell functions triggered by neuroinvasive FNV are different to those triggered by $17 \mathrm{D}$ is currently under investigation in our laboratory, in order to characterize the molecular mechanisms used by each virus to cross the BBB.

We also demonstrated that 17D and FNV were able to infect, replicate and multiply in the Minibrain device. It has previously been described that FNV has an inherently less stable genome than 17D, which could account for the neurovirulent post-vaccine adverse events reported in vaccinees [22]. This leads us to hypothesize that the FNV viruses crossing the BBB in our system could be genetically different from the overall original population [15, 46]. The transcriptomic studies undertaken showed that the 17D and FNV virions crossing the BBB induced specific molecular signatures, and alter the neuronal cell biology differently. These results pave the way for the development of a companion test, and also offer the possibility to study very neatly the quasispecies which can be responsible for these phenotypes.

The final goal of this work is to develop a scientifically relevant test to substitute the "release neurovirulence test" performed in animal (non-human primates and mice) on master seed lot for YFV vaccine. Here we have achieved a pilot study and several steps are still needed before substituting the animal test with this one; in particular, by comparing results obtained in the in vivo and in cellulo assays. Presently, the biological relevance of the biomarkers identified by transcriptome assays is undertaken through in vivo studies. 
The final set up of the in cellulo neurovirulence test will combine viral infection measurement and sensitive neurovirulence marker detection assays. Next steps development will be to extend the validation of this system to other viruses that can cross the $\mathrm{BBB}$, for example, Japanese Encephalitis virus.

In summary, this pilot study has demonstrated that the BBB-Minibrain is an in cellulo test capable of discriminating neuroinvasiveness/neurovirulence of two YF vaccines, in addition it was able to identify the molecular signature of the two YF strains according to their neurovirulence. We consequently propose that the human BBB-Minibrain device could be a predictive in cellulo test for the safety evaluation of live YFV vaccines, for the assessment of neuroinvasiveness and neurotropism. It represents an elegant and promising alternative test to the animal neurovirulent tests in current use $[54,55]$. From a regulatory point of view, significant work has yet to be completed before this system can replace the in vivo monkey test [56]. Nevertheless, this work should be viewed as a positive step in the search for appropriate alternatives to animal testing, in accordance with the principles of the $3 \mathrm{Rs}$ and highlights the power of coordinated regulatory research for the sake of biomedical research in the vaccine field [57]. 


\section{Acknowledgements}

We would like to thank Eric Abachin, Nathalie Mantel, Erika Navarro-Sanchez, Florence Miller, Philippe Afonso, Pierre-Emmanuel Ceccaldi for fruitful discussions. Marie-Pascale Frenkiel and Audrey Chareyre for excellent technical assistance. We are also indebted to Amadou Sall for the gift of the YFV-FNV strain and to Fabrice Agou for access to the fluorescence spectrophotometer Tecan Infinite F500 (USA). Affymetrix experiments have been undertaken with the help of Christelle Thibault-Plateforme GenomEast of IGBMC (Strasbourg, France). Editorial assistance with the preparation of the manuscript was provided by Rebecca Hornby, inScience Communications, Springer Healthcare. Funding for this assistance was provided by Sanofi Pasteur.

\section{Declaration of Interests}

Anaelle Da Costa, Nolwenn Nougarède and Aure Saulnier are employees of Sanofi Pasteur. Cécile Khou, Nathalie Pardigon, Christophe Prehaud and Monique Lafon are employees of Institut Pasteur (Paris).

\section{Author Contributions}

All authors were involved in the concept and design of the study, contributed to the interpretation of the data, participated in the preparation of this manuscript, and approved the final manuscript for submission. All authors had access to the study data and are responsible for the veracity and completeness of the data reported.

\section{Role of Funding Source}

This study and the fellowship of A. da Costa were funded by a grant "Contrat de Soutien à la Recherche" provided by Sanofi Pasteur to Institut Pasteur. 


\section{References}

[1] Salvatico C, Specht CG, Triller A. Synaptic receptor dynamics: from theoretical concepts to deep quantification and chemistry in cellulo. Neuropharmacology. 2015;88:2-9.

[2] Basu B. Biomaterials Science and Tissue Engineering: Principles and Methods. Cambridge: Cambridge University Press; 2017.

[3] Minor PD. Live attenuated vaccines: Historical successes and current challenges. Virology. 2015;479-480:379-92.

[4] Jones PG, Cowan G, Gravendyck M, Nagata T, Robinson S, Waits M. Regulatory requirements for vaccine authorisation. Rev Sci Tech. 2007;26:379-93.

[5] Marshall V, Baylor NW. Food and Drug Administration regulation and evaluation of vaccines. Pediatrics. 2011;127 Suppl 1:S23-30.

[6] WHO. Recommendations to assure the quality, safety and efficacy of live attenuated yellow fever vaccines. Technical Report serie. 2012;978

[7] 0537 M. Yellow Fever Vaccine. European Pharmacopoeia 2013.

[8] Wells DJ. Animal welfare and the 3Rs in European biomedical research. Ann N Y Acad Sci. 2011;1245:14-6.

[9] Caloni F, Costa P, Dal Negro G, De Angelis I, Dotti S, Lazzari G, et al. Alternative methods: 3Rs, research and regulatory aspects. ALTEX. 2013;30:378-80.

[10] Daneshian M, Akbarsha MA, Blaauboer B, Caloni F, Cosson P, Curren R, et al. A framework program for the teaching of alternative methods (replacement, reduction, refinement) to animal experimentation. ALTEX. 2011;28:341-52.

[11] Whittall $\mathrm{H}$. Information on the $3 \mathrm{Rs}$ in animal research publications is crucial. Am $\mathbf{J}$ Bioeth. 2009;9:60-1. 
[12] Niemi SM, Davies GF. Animal Research, the 3Rs, and the "Internet of Things": Opportunities and Oversight in International Pharmaceutical Development. ILAR J. 2016;57:246-53.

[13] Neal JW. Flaviviruses are neurotropic, but how do they invade the CNS? J Infect. 2014;69:203-15.

[14] Swanson PA, 2nd, McGavern DB. Viral diseases of the central nervous system. Curr Opin Virol. 2015;11:44-54.

[15] Vignuzzi M, Stone JK, Arnold JJ, Cameron CE, Andino R. Quasispecies diversity determines pathogenesis through cooperative interactions in a viral population. Nature. 2006;439:344-8.

[16] Chakraborty S, Nazmi A, Dutta K, Basu A. Neurons under viral attack: victims or warriors? Neurochem Int. 2010;56:727-35.

[17] Prehaud C, Megret F, Lafage M, Lafon M. Virus infection switches TLR-3-positive human neurons to become strong producers of beta interferon. J Virol. 2005;79:12893-904.

[18] Chow BW, Gu C. The molecular constituents of the blood-brain barrier. Trends Neurosci. 2015;38:598-608.

[19] Obermeier B, Daneman R, Ransohoff RM. Development, maintenance and disruption of the blood-brain barrier. Nat Med. 2013;19:1584-96.

[20] Spindler KR, Hsu TH. Viral disruption of the blood-brain barrier. Trends Microbiol. 2012;20:282-90.

[21] Duque S, Joussemet B, Riviere C, Marais T, Dubreil L, Douar AM, et al. Intravenous administration of self-complementary AAV9 enables transgene delivery to adult motor neurons. Mol Ther. 2009;17:1187-96. 
[22] Holbrook MR, Li L, Suderman MT, Wang H, Barrett AD. The French neurotropic vaccine strain of yellow fever virus accumulates mutations slowly during passage in cell culture. Virus Res. 2000;69:31-9.

[23] Staples JE, Monath TP. Yellow fever: 100 years of discovery. JAMA. 2008;300:960-2.

[24] Barrett ADT. Yellow fever live attenuated vaccine: A very successful live attenuated vaccine but still we have problems controlling the disease. Vaccine. 2017;35:5951-5.

[25] Theiler M, Smith HH. The Use of Yellow Fever Virus Modified by in Vitro Cultivation for Human Immunization. J Exp Med. 1937;65:787-800.

[26] Weksler BB, Subileau EA, Perriere N, Charneau P, Holloway K, Leveque M, et al. Blood-brain barrier-specific properties of a human adult brain endothelial cell line. FASEB J. 2005;19:1872-4.

[27] Lafon M, Prehaud C, Megret F, Lafage M, Mouillot G, Roa M, et al. Modulation of HLA-G expression in human neural cells after neurotropic viral infections. J Virol. 2005;79:15226-37.

[28] Paquet-Durand F, Tan S, Bicker G. Turning teratocarcinoma cells into neurons: rapid differentiation of NT-2 cells in floating spheres. Brain Res Dev Brain Res. 2003;142:161-7.

[29] Cheung WM, Fu WY, Hui WS, Ip NY. Production of human CNS neurons from embryonal carcinoma cells using a cell aggregation method. Biotechniques. 1999;26:946-8, $50-2,54$

[30] Janabi N, Peudenier S, Heron B, Ng KH, Tardieu M. Establishment of human microglial cell lines after transfection of primary cultures of embryonic microglial cells with the SV40 large T antigen. Neurosci Lett. 1995;195:105-8.

[31] Garcia-Mesa Y, Jay TR, Checkley MA, Luttge B, Dobrowolski C, Valadkhan S, et al. Immortalization of primary microglia: a new platform to study HIV regulation in the central nervous system. J Neurovirol. 2017;23:47-66. 
[32] Siflinger-Birnboim A, Del Vecchio PJ, Cooper JA, Blumenstock FA, Shepard JM, Malik

AB. Molecular sieving characteristics of the cultured endothelial monolayer. J Cell Physiol. $1987 ; 132: 111-7$.

[33] De Bock M, Culot M, Wang N, da Costa A, Decrock E, Bol M, et al. Low extracellular $\mathrm{Ca} 2+$ conditions induce an increase in brain endothelial permeability that involves intercellular Ca2+ waves. Brain Res. 2012;1487:78-87.

[34] Schultz L, Zurich MG, Culot M, da Costa A, Landry C, Bellwon P, et al. Evaluation of drug-induced neurotoxicity based on metabolomics, proteomics and electrical activity measurements in complementary CNS in vitro models. Toxicol In Vitro. 2015;30:138-65.

[35] Rastogi M, Sharma N, Singh SK. Flavivirus NS1: a multifaceted enigmatic viral protein. Virol J. 2016;13:131.

[36] Mukhopadhyay S, Kuhn RJ, Rossmann MG. A structural perspective of the flavivirus life cycle. Nat Rev Microbiol. 2005;3:13-22.

[37] Lin MY, Wang YL, Wu WL, Wolseley V, Tsai MT, Radic V, et al. Zika Virus Infects Intermediate Progenitor Cells and Post-mitotic Committed Neurons in Human Fetal Brain Tissues. Sci Rep. 2017;7:14883.

[38] Barrett AD, Gould EA. Comparison of neurovirulence of different strains of yellow fever virus in mice. J Gen Virol. 1986;67 ( Pt 4):631-7.

[39] Receveur MC, Bruyand M, Pistone T, Malvy D. [Yellow fever vaccination: update on rare and severe adverse effects]. Med Mal Infect. 2009;39:234-41.

[40] Li H, Xing B, Quan Y, Sun M. [The effect of selective phosphatase inhibitors Salubrinal on autophagy and apoptosis in the lung tissue of rats with acute paraquat poisoning]. Zhonghua Wei Zhong Bing Ji Jiu Yi Xue. 2014;26:671-5.

[41] Wang Z, Shi XY, Yin J, Zuo G, Zhang J, Chen G. Role of autophagy in early brain injury after experimental subarachnoid hemorrhage. J Mol Neurosci. 2012;46:192-202. 
[42] Gaucher D, Therrien R, Kettaf N, Angermann BR, Boucher G, Filali-Mouhim A, et al. Yellow fever vaccine induces integrated multilineage and polyfunctional immune responses. J Exp Med. 2008;205:3119-31.

[43] Querec TD, Akondy RS, Lee EK, Cao W, Nakaya HI, Teuwen D, et al. Systems biology approach predicts immunogenicity of the yellow fever vaccine in humans. Nat Immunol. 2009;10:116-25.

[44] Cottin P, Niedrig M, Domingo C. Safety profile of the yellow fever vaccine Stamaril(R): a 17-year review. Expert Rev Vaccines. 2013;12:1351-68.

[45] Monath TP. Yellow fever vaccine. Expert Rev Vaccines. 2005;4:553-74.

[46] Beck A, Tesh RB, Wood TG, Widen SG, Ryman KD, Barrett AD. Comparison of the live attenuated yellow fever vaccine 17D-204 strain to its virulent parental strain Asibi by deep sequencing. J Infect Dis. 2014;209:334-44.

[47] Minor PD. Neurovirulence tests of three 17D yellow fever vaccine strains. Biologicals. 2011;39:167-70.

[48] Fox JP. Immunity to Yellow Fever Encephalitis of Monkeys and Mice Immunized by Neural and Extraneural Routes. J Exp Med. 1943;77:487-506.

[49] Levenbook IS, Pelleu LJ, Elisberg BL. The monkey safety test for neurovirulence of yellow fever vaccines: the utility of quantitative clinical evaluation and histological examination. J Biol Stand. 1987;15:305-13.

[50] Sneddon LU. Pain in laboratory animals: A possible confounding factor? Altern Lab Anim. 2017;45:161-4.

[51] Sneddon LU, Halsey LG, Bury NR. Considering aspects of the 3Rs principles within experimental animal biology. J Exp Biol. 2017;220:3007-16.

[52] Weksler B, Romero IA, Couraud PO. The hCMEC/D3 cell line as a model of the human blood brain barrier. Fluids Barriers CNS. 2013;10:16. 
[53] Mateo RI, Xiao SY, Travassos da Rosa AP, Lei H, Guzman H, Lu L, et al. Yellow fever 17-D vaccine is neurotropic and produces encephalitis in immunosuppressed hamsters. Am $\mathbf{J}$ Trop Med Hyg. 2007;77:919-24.

[54] Semple BD, Blomgren K, Gimlin K, Ferriero DM, Noble-Haeusslein LJ. Brain development in rodents and humans: Identifying benchmarks of maturation and vulnerability to injury across species. Prog Neurobiol. 2013;106-107:1-16.

[55] Monath TP, Myers GA, Beck RA, Knauber M, Scappaticci K, Pullano T, et al. Safety testing for neurovirulence of novel live, attenuated flavivirus vaccines: infant mice provide an accurate surrogate for the test in monkeys. Biologicals. 2005;33:131-44.

[56] Wood D. Neurovirulence. Dev Biol Stand. 1999;101:127-9.

[57] Ramachandra R. Collaborative vaccine development: partnering pays. Hum Vaccin. 2008;4:88-90. 


\section{Figure legends}

\section{Figure 1. Amplification of YF virus in the BBB-Minibrain device}

A. Schematic diagram: The BBB-Minibrain device is composed of two compartments: the luminal compartment with human endothelial cells (hCMEC/D3) mimicking the "blood" area, and the abluminal compartment with human neuron-astrocyte (NT2-N/A) and microglial (CHME) cells mimicking the "brain" area of a human BBB. Right graph: The permeability of hCMEC/D3 cells, measured by the crossing of Lucifer yellow $\left(\mathrm{Pe}_{\mathrm{Ly}}\right)$ after 6 days of culture is not altered in the presence of Minibrain, regardless of the time point. $1 \mathrm{~d}=$ cells put in coculture at day 1 post-seeding.

B. Schematic diagram: Culture of hCMEC/D3 on insert. Right panel: Vero cells are infected by the YF viruses (17D and FNV, a-b anti-Flavivirus E protein antibody, e-f antiYFV NS1 protein), hCMEC/D3 infected by the YF viruses (17D and FNV, c-d antiFlavivirus E protein antibody, $\mathrm{g}-\mathrm{h}$ anti-YFV NS1 protein). Cells are also stained with HCS CellMask Deep Red Stain to visualize the plasma membranes. Nuclei are in blue, E-protein is

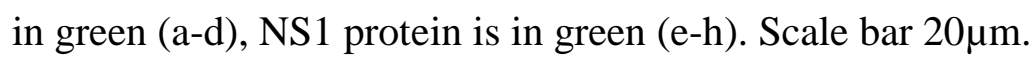

C. Schematic diagram: Culture of Minibrain cells in the abluminal "brain" compartment. Right graph: Minibrain cells express specific markers of neuronal cells: Neurons (NEFH: neurofilament protein $\mathrm{H}$, TH: tyrosine hydroxylase), Astrocytes (AQP4: aquaporin 4, PYGB: glycogen phosphorylase B, GFAP: glial fibrillary astrocytic protein), Microglial cells (CD200R: CD200 receptor) as determined by RT-PCR. Caco2 cells were used as negative control.

D. Left panel: Immunofluorescence images of an infected neuron by 17D (a) or FNV (b) 48h post-infection (NEFH in green, nucleus in blue, virus in red), scale bar $10 \mu \mathrm{m}$, the viral antigens are accumulated as a big spot (white square in a \& b, enlarged in c \& d). Both YF 
strains can infect the neurons in the BBB-Minibrain device but FNV has a stronger fitness.

Right panel, upper panel: Percentage of infected neurons in the culture. Statistical test: Student's t test two-tailed. Cells $n>370$. Right panel, lower graph: Equivalent virus production of 17D and FNV on the cells constituting the abluminal "brain" compartment. Titers are expressed as plaque forming unit per milliliter (pfu/ml).

Figure 2. The BBB-Minibrain device can distinguish the neuroinvasive and neurotropic phenotypes of the two YF strains

A: Schematic diagram of in cellulo testing for neuroinvasiveness. The viruses are added in the luminal compartment and $24 \mathrm{~h}$ later the virions which have crossed the hCMEC/D3 barrier are titrated from the abluminal compartment. B: FNV is more neuroinvasive than the 17D vaccine strain, Students's t test two-tailed. C: Schematic diagram for testing for neuroinvasiveness and neurotropic phenotypes. The viruses are added in the luminal compartment and either 24 or $72 \mathrm{~h}$ later the virions, which have crossed the hCMEC/D3 barrier, are titrated from the abluminal compartment. At the time point $(72 \mathrm{~h})$, the viral genome is quantified by digital droplet PCR. D: At 24 h, FNV is clearly more neuroinvasive than 17D in the BBB-Minibrain device, Students's t test two-tailed; and E: both YF-17D and YF-FNV virions, which have crossed the hCMEC/D3 physiological barrier, are amplified in the Minibrain cells at the $72 \mathrm{~h}$ time point, Students's t test two-tailed. F: Multiplication of the YF-17D and YF-FNV virions are confirmed by the titer in genome equivalent $(72 \mathrm{~h})$, Students's t test two-tailed. 


\section{Figure 3. 17D and FNV differently modify the endothelial cell transcriptome}

Transciptome analysis was carried out on 17D and FNV infected hCMEC/D3 endothelial cells $24 \mathrm{~h}$ post infection (Figures 2A-B). The transcriptomic analyses were undertaken on Affymetrix Gene Chips covering the entire human genome (U133 2.0 Plus).

A: Venn diagram of the genes with modified expression in response to 17D (yellow) or FNV (blue), compared with the sham control. 17D up-regulates 37 genes and FNV 57. 18 genes are common between 17D and FNV, and both strains do not down regulate any gene expression. B: Gene identity and fold expression change. 17D and FNV have specific molecular signatures with some genes in common. C: Intuitive Pathway Analysis analysis of the genes up-regulated by 17D and FNV.

\section{Figure 4. 17D and FNV have distinct molecular signatures in the BBB-Minibrain device}

A transcriptome analysis was carried out on the infected cerebral cellular extracts presented Fig $2 \mathrm{C}-\mathrm{F}$ at the time point $72 \mathrm{~h}$. The transcriptomic analyses were undertaken on Affymetrix Gene Chip covering the entire human genome (U133 2.0 Plus).

A: Venn diagram of the genes which expression is modified by 17D (yellow) or FNV (blue) compared to the sham control. 17D up-regulate 30 genes and FNV 58. No genes are in common between 17D and FNV and both strains never down regulate any gene expression. B: Gene identity and fold expression change. The genes in red are genes which have been involved in hepatotoxicity in a liver context. 17D and FNV have each a specific molecular signature. The whole in part diagram showed the overall distribution of the genes for 17D (yellow colors) and FNV (blue colors) based on their fold change. C: Intuitive Pathway Analysis of the genes up-regulated by 17D and FNV infections. In the Minibrain cells, 17D infection is mainly affecting cell organization (movement, development, signaling and cell- 
cell interaction). On the contrary, FNV infection affects cellular biochemistry and metabolism. 